Moreno Santana, Ana; Axpe Caballero, Ángeles; Acosta Rodríguez, Víctor (2012). Efectos de un programa de intervención en el lenguaje sobre el desarrollo del léxico y del procesamiento fonológico en escolares de Educación Infantil con Trastorno Específico del Lenguaje. Revista de Investigación Educativa, 30 (1), 71-86.

\title{
EFECTOS DE UN PROGRAMA DE INTERVENCIÓN EN EL LENGUAJE SOBRE EL DESARROLLO DEL LÉXICOY DEL PROCESAMIENTO FONOLÓGICO EN ESCOLARES DE EDUCACIÓN INFANTIL CON TRASTORNO ESPECÍFICO DEL LENGUAJE'
}

\author{
Ana Moreno Santana \\ Ángeles Axpe Caballero \\ Víctor Acosta Rodríguez \\ Dpto. de Didáctica e Investigación Educativa
}

\section{RESUMEN}

Este estudio muestra algunos resultados de una investigación realizada con escolares con Trastorno Específico del Lenguaje (TEL). Su objetivo fue diseñar un programa de intervención basado en los principios de la educación inclusiva, que apoyara el desarrollo del lenguaje y el acceso a la lectura con una muestra de seis escolares de Educación Infantil, por medio de una serie de instrumentos que median el nivel de desarrollo lingüístico y cognitivo. La aplicación se realizó en los Colegios a los que acudían los niños, combinándose una fase en el aula habitual con otra en el aula de logopedia. En este trabajo mostramos los efectos del programa en las dimensiones relativas al desarrollo del léxico y del procesamiento fonológico. Se efectuó un análisis de contraste pretest-postest en las variables de léxico, procesamiento fonológico y longitud de enunciados. Los resultados reflejan avances significativos en prácticamente todas las variables analizadas.

\footnotetext{
Correspondencia:

Ana Moreno Santana. ammoreno@ull.es

E-mail autores: Ángeles Axpe (aaxpe@ull.es), Víctor Acosta (vacosta@ull.es).

1 Agradecemos la ayuda recibida a través del Proyecto de Investigación La intervención integrada en el lenguaje y la lectura emergente con niños con Trastorno Específico de Lenguaje (TEL), financiado por el Ministerio de Ciencia e Innovación con referencia SEJ2007-67037/EDUC
} 
Palabras clave: Educación inclusiva; Intervención temprana; Léxico; Trastorno específico del lenguaje; Procesamiento fonológico.

\title{
EFFECTS OF A LANGUAGE INTERVENTION PROGRAMME ON THE DEVE- LOPMENT OF LEXIS AND PHONOLOGICAL PROCESSING IN CHILDHOOD EDUCATION PUPILS WITH SPECIFIC LANGUAGE IMPAIRMENTS
}

\begin{abstract}
This study presents some of the results of a research study on Specific Language Impairment (SLI) pupils. Our aim was to design an intervention programme based on inclusive education principles, which could support both language development and access to reading of a sample. The sample used was made up of six SLI children who had been diagnosed with the help of language and cognitive tests. Implementation was carried out in the pupils' schools during regular classroom hours as well during remedial classes. In this study we show the effects of the programme on lexical development and phonological processes. The contrast between pre and post-tests was analysed for variables such as lexis, phonological processing and length of utterances. The results show significant improvements in all variables under study.

Key words: Early intervention; Inclusive education; Phonological processing; Specific language impairment; Vocabulary.
\end{abstract}

\section{INTRODUCCIÓN}

La mejor escolarización y respuesta educativa que precisa el alumnado con TEL es un tema no resuelto todavía. Hay autores y experiencias (Conti Ramsden y Botting, 2000) que señalan que las características de este trastorno hace que este alumnado se beneficie más de la enseñanza proporcionada en las llamadas Unidades de Lenguaje (Reino Unido), incluso en otros casos (por ejemplo Chile) existen colegios específicos para niños y niñas con TEL como primer acceso a la escolarización.

En el contexto español, las últimas reformas y propuestas educativas apuestan decididamente por la inclusión como respuesta que busca el logro progresivo de la calidad y equidad de todo el alumnado (LOE, 2006). Ese nuevo reto ha dado lugar a investigaciones cuyo objetivo es analizar la realidad y buscar fórmulas y estrategias didácticas que contribuyan al logro de dicha meta (Cardona, 2003). Ocurre, sin embargo que los avances experimentados en la atención a las necesidades educativas singulares del alumnado ha sido desigual en razón, muchas veces, del conocimiento existente del propio trastorno y de la dificultad intrínseca del mismo (Fernández et al., 2001). El TEL es un buen reflejo de ello, y eso explica que, en general, suela identificarse tarde en las escuelas, cuando ya los niños han consolidado un cúmulo de disfunciones y alteraciones que en algunos casos sobrepasa lo puramente lingüístico y trasciende al plano social. Las características propias del trastorno, como son su escasa incidencia (alrededor del 7'4\% de la población infantil, Tombling et al., 1997), su enorme heterogeneidad, la ausencia de déficits cognitivos, socioambientales, sensoriales y neurológicos, junto con graves problemas a la hora de acceder y desarrollar el lenguaje oral en todos 
sus componentes, justifican la dificultad para detectarlos tempranamente. Ante esta evidencia, en trabajos anteriores hemos planteado la necesidad de diseñar modelos de evaluación e intervención que partan de los presupuestos de la educación inclusiva, al objeto de afrontar esta problemática (Acosta, et al., 2007; Acosta, et al., 2008). En esa línea debe entenderse el estudio que presentamos. El fundamento teórico adoptado se sostiene en los modelos anglosajones, recogidos en el Acta para la Educación de los Individuos con Discapacidad (IDEA, 2004), donde se plantea una intervención secuenciada en 3 niveles, como procedimiento para garantizar la inclusión de los escolares con diferentes necesidades educativas. En el nivel 1, el tutor, con el asesoramiento y apoyo del logopeda, trabaja con todos los niños en el aula habitual, aunque haciendo un mayor seguimiento a aquellos que presentan condiciones de riesgo. Se persigue con ello identificar al alumnado que no progrese dentro del aula, al objeto de proporcionarles una intervención más intensa y frecuente modificando, si fuera necesario, la organización habitual del aula (nivel 2). Se trata, finalmente, de cumplir con el objetivo de la detección temprana identificando sujetos de riesgo y, en definitiva, comenzar a trabajar desde la prevención. En los casos en que el progreso no sea el esperado, se intensifica el apoyo en el nivel 3 (Bradley, et al., 2005; Justice, 2006).

De forma concreta, nuestro estudio ha partido de esos planteamientos, pretendiendo dos objetivos fundamentales. En primer lugar, detectar tempranamente a escolares con el perfil que caracteriza al TEL, aprovechando la corta edad de acceso a la escolarización en nuestro país. El segundo objetivo fue elaborar una propuesta de acción educativa inclusiva, que apoyara el proceso de aprendizaje de esos escolares en el terreno del lenguaje y de la lectura inicial. En este artículo presentaremos los resultados obtenidos tras dos periodos de trabajo (enero-junio del 2009 y octubre de 2009 a mayo de 2010) referidos a la evolución en los ámbitos relacionados con el léxico y el procesamiento fonológico. No obstante, este trabajo forma parte de una investigación más amplia que estudia otros aspectos, como son la influencia de los contextos familiar y escolar, analizando el perfil y prácticas que tienen lugar en esos escenarios, y otro tipo de variables en el TEL, tales como el desarrollo narrativo, cognitivo, etc.

\section{UN MODELO DE INTERVENCIÓN EN NIVELES}

La propuesta de trabajo de intervención en niveles está pensada para actuar desde la etapa de Educación Infantil, antes de que los fracasos se consoliden y se encadenen unos con otros. Por lo tanto, persigue apoyar las necesidades educativas del alumnado desde la prevención (Fuchs y Fuchs, 2006; Vaughn, et al., 2007), contemplándose varios niveles de enseñanza (Acosta, et al., en prensa)

En nuestra investigación hemos considerado dos de los tres niveles, en concreto los niveles 2 y 3, los cuales describiremos brevemente.

El nivel 2 de trabajo se caracteriza por proporcionar una enseñanza intensiva, mediante la estimulación focalizada desde el escenario del aula. El trabajo es de naturaleza colaborativa, siendo la labor del especialista la de coordinar su acción con el profesor para que ambos actúen conjuntamente en la implementación del programa, recurriendo a estrategias basadas en el uso del apoyo y andamiaje (Coleman, et al., 2006). La frecuencia e intensidad del trabajo en este nivel han sido muy variables a la 
hora de aplicarlo con sujetos de Educación Infantil. Los extremos se sitúan en los 10-15 minutos durante 3 días a la semana (O’Connor, et al., 2005) hasta los 30-45 minutos durante 5 días a la semana (Simmons et al., 2008).

El nivel 3 está previsto para aquellos que no progresen adecuadamente en la situación anterior. Lo caracteriza su mayor intensidad, así como su aplicación individual por parte del logopeda y, si se considera necesario, fuera del aula habitual del niño (Vaughn, et al., 2003).

Así pues, continuando con la línea de reflexión ya iniciada por nuestro grupo en trabajos anteriores sobre habilidades de lenguaje oral y lectura inicial en sujetos con TEL (Acosta, et al., 2008; Acosta, et al., a y b, en prensa), nos propusimos en esta ocasión diseñar y evaluar la efectividad de un programa de intervención para niños con TEL de 4 años, organizado con la secuencia y contenidos propios de los niveles 2 y 3 .

\section{OBJETIVOS}

El objetivo del estudio fue evaluar el alcance de un programa de intervención de naturaleza inclusiva en los niveles 2 y 3 de ejecución, con el cual se perseguía mejorar el lenguaje y las habilidades de lectura inicial en niños con TEL. El trabajo se desarrolló en dos periodos, que en total ocuparon 14 meses. El artículo pretende responder a dos interrogantes:

1. ¿Un trabajo inclusivo en los niveles 2 y 3 de intervención puede mejorar ciertas habilidades de lenguaje oral y de lectura inicial en niños con TEL, y más concretamente las referidas al procesamiento fonológico?

2. ¿Qué efecto se produce sobre el desarrollo léxico y la longitud de las oraciones?

\section{METODOLOGÍA}

\section{I.Selección de la muestra: procedimiento, criterios e instrumentos}

Aulas. El proceso de selección de la muestra comenzó aplicando un cuestionario a un amplio sector del profesorado de Educación Infantil de 3 años, que nos permitió obtener información de 400 escolares en aspectos relacionados con la audición, el lenguaje y el habla (Acosta, et al., 2007).

Esta primera criba arrojó un total de 32 escolares que parecían tener algún tipo de dificultad, a los cuales se les administraron tres pruebas (Test de Vocabulario en Imágenes Peabody, Dunn et al., 1986; Inventario de Desarrollo Battelle, Newborg, et al., 2001; Prueba de Lenguaje Oral de Navarra-Revisada, Aguinaga et al., 2004). Posteriormente se procedió a un proceso de verificación de que el perfil lingüístico correspondía con lo establecido para el TEL. El resultado de este proceso conformó la muestra final, formada por 9 niños, distribuidos en 8 colegios públicos diferentes. De ellos, 6 constituyeron el grupo experimental y los 3 restantes se tomaron como grupo control, no haciéndose ningún trabajo con ellos.

La ratio de las clases seleccionadas oscilaba en un rango que iba desde los 15-22 niños. La investigación comenzó después de debatir el proyecto con los respectivos Equipos Directivos y de Orientación Psicopedagógica de cada uno de los Centros. 


\section{Descripción de la muestra final y perfil lingüístico}

Niños. El proceso de selección inicial arrojó dificultades entre los escolares que iban desde niños con retraso del lenguaje y/o habla y otro grupo que parecían presentar un perfil propio del TEL. La confirmación de este último diagnóstico se obtuvo tras un exhaustivo proceso de evaluación, al objeto de valorar las áreas y procesos de dificultad específicos del TEL. Los resultados se muestran en la tabla I.

TABLA I

INSTRUMENTOS UTILIZADOS Y RESULTADOS DE LA EVALUACIÓN INICIAL PARA DIAGNOSTICAR A LOS SUJETOS CON TEL

\begin{tabular}{|c|c|}
\hline Pruebas & Resultados \\
\hline $\begin{array}{l}\text { Prueba de Lenguaje PLS-4 (Zimmerman, } \\
\text { Steiner y Pond, 2004) }\end{array}$ & $\begin{array}{l}\text { Todos los sujetos estaban entre }-1 \text { y }-2 \\
\text { desviaciones estándar por debajo de la } \\
\text { media }\end{array}$ \\
\hline $\begin{array}{l}\text { Escala de Desarrollo Cognitivo General } \\
\text { (McCarthy, 1976) }\end{array}$ & $\begin{array}{l}\text { Desarrollo General Cognitivo, con un } \\
\text { rango entre } 73 \text { y } 95 \text {. } \\
\text { Todos los sujetos presentaron retraso en } \\
\text { el factor verbal, mientras que el desarro- } \\
\text { llo no verbal era acorde con su edad }\end{array}$ \\
\hline $\begin{array}{l}\text { Registro Fonológico Inducido (Juárez y } \\
\text { Monfort, 1996) }\end{array}$ & $\begin{array}{l}\text { Tanto en fonemas como en palabras erró- } \\
\text { neas puntuación muy por debajo de la } \\
\text { mínima establecida por el test. }\end{array}$ \\
\hline $\begin{array}{l}\text { Prueba de Discriminación Auditiva } \\
\text { (Aguilar y Serra, 2003) }\end{array}$ & Por debajo del percentil 50 \\
\hline Pseudopalabras (Aguado, 2007) & $\begin{array}{l}\text { Resultados muy bajos tanto en palabras } \\
\text { familiares como en no familiares }\end{array}$ \\
\hline $\begin{array}{l}\text { Imitación de frases (WPPSI, Wechsler, } \\
\text { 1970) }\end{array}$ & $\begin{array}{l}\text { Puntuaciones entre el rango } 1 \text { y } 10 \text {, muy } \\
\text { por debajo de la puntuación inferior del } \\
\text { test (11) }\end{array}$ \\
\hline Semejanzas (WPPSI, Wechsler, 1970) & $\begin{array}{l}\text { Puntuaciones entre el rango } 0 \text { y } 6 \text {, muy } \\
\text { por debajo de la puntuación inferior del } \\
\text { test (10) }\end{array}$ \\
\hline Vocabulario (WPPSI, Wechsler, 1970) & $\begin{array}{l}\text { Puntuaciones entre el rango } 0 \text { y } 9 \text {, muy } \\
\text { por debajo de la puntuación inferior del } \\
\text { test (10) }\end{array}$ \\
\hline $\begin{array}{l}\text { Asociación Visual (ITPA, Kirk, McCarty } \\
\text { y Kirk, 1968) }\end{array}$ & $\begin{array}{l}\text { Puntuaciones entre el rango } 1 \text { y } 10 \text {, muy } \\
\text { por debajo del límite inferior del test, es- } \\
\text { tablecido para los } 3 \text { años de edad. }\end{array}$ \\
\hline
\end{tabular}




\begin{tabular}{|c|c|}
\hline Pruebas & Resultados \\
\hline $\begin{array}{l}\text { Asociación Auditiva (ITPA, Kirk, Mc- } \\
\text { Carty y Kirk, 1968) }\end{array}$ & $\begin{array}{l}\text { Puntuaciones entre el rango } 3 \text { y 10, muy } \\
\text { por debajo del límite inferior del test, es- } \\
\text { tablecido para los } 3 \text { años de edad. }\end{array}$ \\
\hline $\begin{array}{l}\text { Memoria secuencial auditiva (ITPA, } \\
\text { Kirk, McCarty y Kirk, 1968) }\end{array}$ & $\begin{array}{l}\text { Puntuaciones entre el rango } 1 \text { y } 4 \text {, muy } \\
\text { por debajo del límite inferior del test, es- } \\
\text { tablecido para los } 3 \text { años de edad. }\end{array}$ \\
\hline $\begin{array}{l}\text { Evaluación de Guiones (Pavez, Coloma } \\
\text { y Maggiolo, 2008) } \\
\text { Desarrollo narrativo (comprensión y } \\
\text { producción del cuento Buenas noches, go- } \\
\text { rila; Peggy, 2001) }\end{array}$ & $\begin{array}{l}\text { - Las puntuaciones oscilan entre las de } 4 \\
\text { niños que estarían dentro del promedio } \\
\text { y otros } 5 \text { que tendrían un desempeño } \\
\text { descendido (entre }-1,5 \text { y -2 desviaciones } \\
\text { estándar) } \\
\text {-Déficit narrativo en comprensión y pro- } \\
\text { ducción }\end{array}$ \\
\hline $\begin{array}{l}\text { Procesamiento fonológico (Jiménez y } \\
\text { Ortiz,1995) }\end{array}$ & $\begin{array}{l}\text { Puntuaciones entre los centiles 1-5 } \\
\text { Puntuación Hepta: muy bajo }\end{array}$ \\
\hline $\begin{array}{l}\text { Procesamiento fonológico-PROFON } \\
\text { (Lara, Aguilar y Serra, 2007) }\end{array}$ & $\begin{array}{l}\text {-En los tres niveles de conciencia fono- } \\
\text { lógica (silábico, intrasilábico y fonémico) } \\
\text { no se supera la puntuación cero. } \\
\text {-En el conocimiento del nombre de las } \\
\text { letras, puntuaciones entre el rango } 0 \text { y } 5 \\
\text { En el conocimiento del sonido de las le- } \\
\text { tras, puntuaciones entre el rango } 0 \text { y } 5\end{array}$ \\
\hline Análisis morfo-léxico & $\begin{array}{l}\text { Diversidad de palabras: rango entre } 16 \\
\text { y } 31 \\
\text { Longitud media de enunciados (LME): } \\
\text { rango entre } 0,7 \text { y } 1,5\end{array}$ \\
\hline
\end{tabular}

Como se desprende de estos datos, el perfil obtenido concuerda con las características del TEL, al comprobarse que el lenguaje y los procesos psicolingüísticos básicos se encuentran seriamente afectados. Más en concreto, por lo que respecta al objetivo de este estudio, conviene destacar las puntuaciones iniciales en las pruebas que examinaban el nivel de desarrollo léxico y de procesamiento fonológico. Con respecto al léxico, los subtest del WPPSI referidos a vocabulario y semejanzas partían de unos resultados que situaban las puntuaciones en los rangos 0 y 9 , para el primer caso, y en los rangos 0 y 6 para el segundo; siendo las puntuaciones directas mínimas establecidas para esas tareas de 11 y 10, respectivamente. Por lo que se refiere al procesamiento fonológico, los resultados en la prueba de Jiménez y Ortiz (1995) situaban a los niños en los centiles más bajos (1-5), con un rendimiento global de la prueba "muy bajo", aspecto que se refrenda también en el PROFON, donde los 
niños no superan la puntuación cero en los tres niveles de conciencia fonológica, y se encuentran entre el rango 0 y 5 tanto en el conocimiento del nombre como del sonido de las letras. Por otro lado, en situaciones no estructuradas de evaluación, usando la muestra de lenguaje (juego interactivo evaluador-niño con una granja durante 15 minutos), el empleo de palabras diferentes (calculada sobre las categorías de sustantivos, verbos, adjetivos y adverbios) (Miller, 1991) se movía en un rango de 16 y 31 palabras y, por último, los valores de Longitud Media de Enunciado, obtenidos en la misma situación anterior estaban entre los rangos de 0,7 y 1,5 antes de iniciarse el programa de intervención. La escasez de trabajos de esta naturaleza nos impide relacionar estos últimos resultados con los hallados en otros estudios; solo a modo de aproximación podemos decir que el valor de la Longitud Media de Enunciado en niños con desarrollo típico suele estar muy cercano a su edad cronológica cuestión que, en nuestro caso comprobamos que se aleja bastante

\section{Descripción del programa de intervención}

\section{Programa de intervención nivel 2}

El trabajo en este nivel se implementó entre los meses de enero y junio de 2009, cuando los niños estaban escolarizados en Educación Infantil de 4 años. Se realizaron 48 sesiones, con una frecuencia de tres veces por semana y una duración de 45 minutos, cada una. Las sesiones tenían lugar en la primera hora de la mañana, coincidiendo con el espacio horario que las profesoras dedicaban al manejo del lenguaje oral (asamblea, rutinas y narración). Durante el desarrollo del programa, en cada aula estaban presentes la profesora y una logopeda, que lideraba la ejecución de la intervención.

En la elaboración del programa se tuvo en cuenta la organización de la enseñanza en cada una de las aulas. Tras una serie de seminarios y talleres conjuntos se procedió al establecimiento de objetivos, contenidos y procedimientos del mismo, y se seleccionaron los materiales necesarios. Cuando el programa fue bien conocido por las logopedas y por las maestras, comenzó su implementación colaborativa en las aulas, realizando un seguimiento periódico mediante la observación directa y entrevistas con las profesoras, al objeto de resolver las dificultades que se iban encontrando y valorar los logros alcanzados.

Los objetivos establecidos con la intervención perseguían el fomento prioritario y regular de habilidades relacionadas con el lenguaje oral y la lectura inicial.

Los contenidos se organizaron siguiendo un patrón de complejidad creciente (op. cit. Acosta, et. al., b, en prensa). En la tabla II se presentan dichos contenidos diferenciados por áreas de trabajo.

Esta modalidad perseguía mejorar aspectos tan importantes como el vocabulario, el desarrollo gramatical, el discurso narrativo y el procesamiento fonológico. 


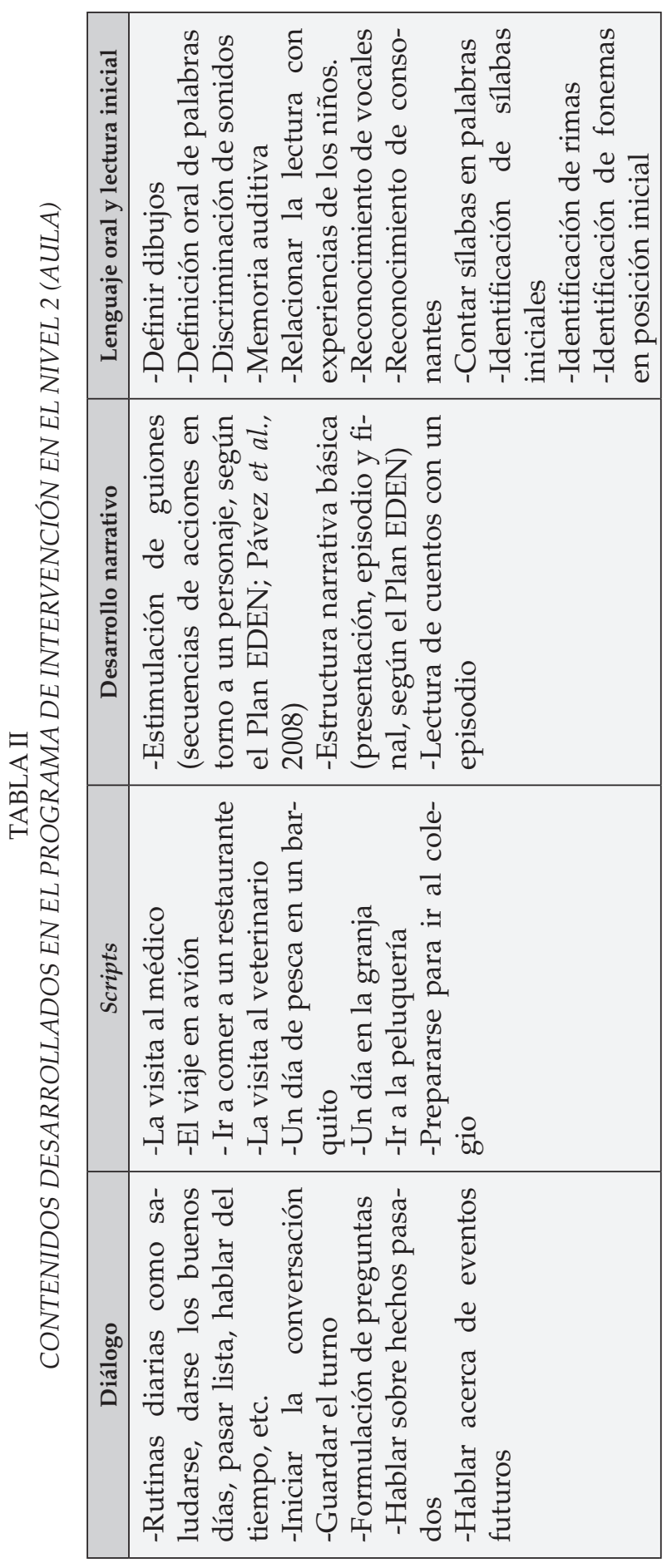

RIE, vol. 30-1 (2012) 


\section{Programa de intervención nivel 3}

Como ya hemos señalado, una segunda fase del programa se basó en el nivel 3. En este caso, se hacía un trabajo individual con el niño con TEL fuera del aula ordinaria durante treinta minutos, aproximadamente. En esta fase se realizaron 72 sesiones, cuando los niños cursaban el último curso de Educación Infantil, con una frecuencia de tres veces por semana. Las características del mismo se corresponden con una aproximación más clínica e intensiva y se redujeron, asimismo, los objetivos de la intervención, focalizándolos hacia el desarrollo del procesamiento y conciencia fonológica (conciencia léxica, silábica y fonémica) y del léxico, por medio del diálogo, scripts y narraciones. Las estrategias o sistemas de facilitación empleados por la logopeda se basaron ahora en los principios de la lectura dialógica, en el recast conversacional y en el modelado interactivo, principalmente.

En la tabla III se presentan los contenidos trabajados en el nivel 3

TABLA III

CONTENIDOS DESARROLLADOS EN EL PROGRAMA DE INTERVENCIÓN EN EL NIVEL 3 (AULA DE LOGOPEDIA)

\begin{tabular}{|c|c|}
\hline $\begin{array}{l}\text { Lenguaje oral a partir } \\
\text { de la estimulación narrativa }\end{array}$ & Procesamiento Fonológico \\
\hline $\begin{array}{l}\text {-Estructura narrativa básica (la presentación, el } \\
\text { episodio y el final, según el Plan EDEN, Pávez } \\
\text { et al., 2008) } \\
\text {-Microestructura narrativa: aspectos gramatica- } \\
\text { les } \\
\text {-Lectura dialógica con andamiaje } \\
\text {-Responde a preguntas que impliquen denomi- } \\
\text { nación de objetos, lugares y personajes de la his- } \\
\text { toria } \\
\text {-Lectura de cuentos con un episodio } \\
\text {-Identifica el personaje principal y expresar algu- } \\
\text { nas de sus características } \\
\text {-Expresa acciones y sentimientos que desencade- } \\
\text { nan la historia } \\
\text {-Recontado de cuento escuchado previamente } \\
\text {-Construye una pequeña historia a partir de un } \\
\text { animal }\end{array}$ & $\begin{array}{l}\text {-Identifica sonidos del ambiente } \\
\text {-Discrimina entre sonidos } \\
\text {-Identifica rimas en canciones } \\
\text {-Recita y completa rimas } \\
\text {-Diferencia palabras largas y cortas en función } \\
\text { del número de sílabas } \\
\text {-Segmenta palabras en las frases } \\
\text {-Identifica objetos que comiencen por la misma } \\
\text { sílaba que el objeto estímulo } \\
\text {-Identifica las sílabas que contiene el nombre de } \\
\text { un objeto } \\
\text {-Discriminar entre objetos cuyo nombre conten- } \\
\text { ga dos o más sílabas } \\
\text {-Identifica la posición de una sílaba concreta (ini- } \\
\text { cial, media, final) en el nombre de varios objetos } \\
\text {-Aisla el fonema vocálico inicial, asociándolo con } \\
\text { su grafía } \\
\text {-Aisla fonemas vocálicos en palabras } \\
\text {-Escribe las vocales contenidas en determinadas } \\
\text { palabras } \\
\text {-Asocia fonemas consonánticos con sus grafías } \\
\text { - Discrimina sonidos consonánticos en palabras } \\
\text {-Une secuencias de grafías aisladas con su pala- } \\
\text { bra }\end{array}$ \\
\hline
\end{tabular}




\section{Análisis de datos}

Con el grupo experimental se realizó un contraste entre los resultados obtenidos en la evaluación inicial y final, una vez aplicado el programa de intervención. Con el grupo control, se seleccionaron cuatro de las pruebas de la evaluación inicial y se volvieron a pasar coincidiendo con la evaluación final. Debido al tamaño reducido de la muestra, en ambos casos realizamos análisis no paramétricos (Camacho, 1994), en concreto, la prueba de rangos de Wilcoxon, sirviéndonos del programa SPSS/PC+ (versión 18.0), para conocer si existían diferencias significativas en las dos evaluaciones en cada uno de los grupos, en las variables seleccionadas.

\section{RESULTADOS}

Para valorar la efectividad del programa, presentamos a continuación los resultados del contraste entre la evaluación inicial y final del grupo experimental, en aquellas variables que informan del rendimiento en el plano del léxico y en aspectos directamente relacionados con las bases del aprendizaje lector (conciencia fonológica y procesamiento fonológico). Con respecto al léxico, los análisis se han hecho comparando las puntuaciones obtenidas en la evaluación inicial y final, tanto con el uso de instrumentos estandarizados, como el Peabody y los subtests de vocabulario y semejanzas del WPPSI, como con las muestras de lenguaje, de donde se han extraído los valores de Longitud Media de Enunciado y el volumen de palabras diferentes (diversidad léxica).

\section{Léxico}

El contraste pretest-postest en las tareas que valoraban el desarrollo léxico comprensivo y expresivo mostró los resultados siguientes:

TABLA IV

CONTRASTE DE LAS VARIABLES DE LÉXICO ANTES Y DESPUÉS DEL PROGRAMA DE INTERVENCIÓN EN EL GRUPO EXPERIMENTAL (MEDIA, DESVIACIÓN TÍPICA Y PRUEBA DE WILCOXON)

\begin{tabular}{|c|c|c|c|}
\hline LÉXICO & EVALUACIÓN INICIAL & EVALUACIÓN FINAL & $\begin{array}{c}\text { SIGNIFICATIVIDAD } \\
\text { PRUEBA DE WILCOXON }\end{array}$ \\
\hline $\begin{array}{c}\text { Peabody (Edad } \\
\text { Equivalente) }\end{array}$ & $\begin{array}{c}\mathrm{M}=2,58 \\
\mathrm{DT}=0,82\end{array}$ & $\begin{array}{c}\mathrm{M}=4,83 \\
\mathrm{DT}=0,70\end{array}$ & $0,028^{*}$ \\
\hline Vocabulario (WPPSI) & $\begin{array}{c}\mathrm{M}=4,00 \\
\mathrm{DT}=3,46\end{array}$ & $\begin{array}{c}\mathrm{M}=13,50 \\
\mathrm{DT}=3,78\end{array}$ & $0,027^{*}$ \\
\hline Semejanzas (WPPSI) & $\mathrm{M}=3,83$ & $\mathrm{M}=11,66$ & $0,026^{*}$ \\
\hline $\begin{array}{c}\text { LME (Longitud Media } \\
\text { de Enunciado) }\end{array}$ & $\mathrm{DT}=2,40$ & $\mathrm{DT}=3,72$ & $0,043^{*}$ \\
\hline $\begin{array}{c}\text { Palabras diferentes } \\
\text { (diversidad léxica) }\end{array}$ & $\begin{array}{c}\mathrm{MT}=25^{\prime} 50 \\
\mathrm{DT}=5,46\end{array}$ & $\begin{array}{c}\mathrm{MT}=3,83 \\
\mathrm{DT}=1,31\end{array}$ & $0,028^{*}$ \\
\hline
\end{tabular}

$*=\mathrm{p} \leq 0.05$ 
Estos resultados permiten ver que, globalmente, los sujetos mejoraron significativamente su desarrollo léxico como consecuencia del trabajo realizado, tanto en el plano comprensivo (reflejado en el aumento de la edad equivalente obtenida en el Peabody) como en el expresivo, donde los niños en la evaluación final fueron capaces de aumentar significativamente el número de objetos que se les pedía definir mediante el subtest de vocabulario del WPPSI, así como de expresar lingüísticamente las semejanzas habidas entre pares de objetos. Con respecto a las situaciones más espontáneas, los datos van en la misma dirección. Se produce un aumento significativo del volumen de palabras (algo más del doble en la evaluación final frente a la inicial) y un incremento en los valores de LME, los cuales pasan de uno a cerca de cuatro (cercano, por lo tanto a su edad cronológica, que en el momento de la evaluación final tenían 5 años).

\section{Procesamiento fonológico}

Los cambios habidos en esta dimensión se valoraron utilizando las pruebas de Conciencia Fonológica de Jiménez y Ortiz (1995) y el Test de Procesamiento FonológicoPROFON (Lara, et al., 2007). Los resultados se presentan en la tabla V.

TABLA V

CONTRASTE EN LAS PRUEBAS DE CONCIENCIA FONOLÓGICA Y PROFON ANTES Y DESPUÉS DE LA INTERVENCIÓN EN EL GRUPO EXPERIMENTAL (MEDIA, DESVIACIÓN TÍPICA Y PRUEBA DE WILCOXON)

\begin{tabular}{|c|c|c|c|}
\hline $\begin{array}{l}\text { PROCESAMIENTO } \\
\text { FONOLÓGICO }\end{array}$ & $\begin{array}{l}\text { EVALUACIÓN } \\
\text { INICIAL }\end{array}$ & EVALUACIÓN FINAL & \begin{tabular}{|c|} 
SIGNIFICATIVIDAD \\
PRUEBA DE WILCOXON
\end{tabular} \\
\hline $\begin{array}{c}\text { Conciencia Fonológica } \\
\text { (p. directa) }\end{array}$ & $\begin{array}{l}\mathrm{M}=7,00 \\
\mathrm{DT}=9,50\end{array}$ & $\begin{array}{l}\mathrm{M}=35,83 \\
\mathrm{DT}=19,18\end{array}$ & $0,027^{*}$ \\
\hline $\begin{array}{l}\text { Conciencia Fonológica } \\
\text { (p. centil) }\end{array}$ & $\begin{array}{l}\mathrm{M}=4,25 \\
\mathrm{DT}=4,28\end{array}$ & $\begin{array}{l}\mathrm{M}=44,25 \\
\mathrm{DT}=30,93\end{array}$ & $0,027^{*}$ \\
\hline $\begin{array}{c}\text { Procesamiento fonológico } \\
\text { (nivel silábico) }\end{array}$ & $\begin{array}{l}\mathrm{M}=1,50 \\
\mathrm{DT}=1,37\end{array}$ & $\begin{array}{l}\mathrm{M}=9,66 \\
\mathrm{DT}=2,80\end{array}$ & $0,027^{*}$ \\
\hline $\begin{array}{c}\text { Procesamiento fonológico } \\
\text { (nivel intrasilábico) }\end{array}$ & $\begin{array}{l}\mathrm{M}=1,83 \\
\mathrm{DT}=2,71\end{array}$ & $\begin{array}{l}\mathrm{M}=5,33 \\
\mathrm{DT}=2,25\end{array}$ & 0,115 \\
\hline $\begin{array}{l}\text { Procesamiento fonológico } \\
\text { (nivel fonémico) }\end{array}$ & $\begin{array}{c}\mathrm{M}=0,83 \\
\mathrm{DT}=0,98\end{array}$ & $\begin{array}{l}\mathrm{M}=4,50 \\
\mathrm{DT}=2,88\end{array}$ & $0,043^{*}$ \\
\hline Memoria fonológica & $\begin{array}{l}M=16,33 \\
\mathrm{DT}=12,62\end{array}$ & $\begin{array}{l}\mathrm{M}=31,50 \\
\mathrm{DT}=6,89\end{array}$ & $0,028^{*}$ \\
\hline $\begin{array}{l}\text { Reconocimiento nombre } \\
\text { letras }\end{array}$ & $\begin{array}{l}\mathrm{M}=2,00 \\
\mathrm{DT}=2,28\end{array}$ & $\begin{array}{l}\mathrm{M}=14,00 \\
\mathrm{DT}=4,38\end{array}$ & $0,028^{*}$ \\
\hline $\begin{array}{c}\text { Reconocimiento sonido } \\
\text { letras }\end{array}$ & $\begin{array}{l}\mathrm{M}=2,00 \\
\mathrm{DT}=2,28\end{array}$ & $\begin{array}{l}\mathrm{M}=10,50 \\
\mathrm{DT}=4,32\end{array}$ & $0,026^{*}$ \\
\hline
\end{tabular}

$*=p \leq 0.05$ 
Los datos reflejan que la parte del programa centrada en procesamiento fonológico produjo igualmente cambios estadísticamente significativos en casi todas las variables analizadas. Así se comprueba en los resultados globales que aporta la prueba de Jiménez y Ortiz (1995), donde se observa una mejora notable en las puntuaciones directas y en los centiles después de la aplicación del programa. Esta ganancia global se corrobora y precisa con los resultados del PROFON. Con ella podemos observar que los mayores beneficios se corresponden con el nivel silábico (conciencia silábica), seguido del nivel fonémico (conciencia fonémica), mientras que el programa no tuvo efectos estadísticamente significativos en el nivel intrasilábico (conciencia intrasilábica).

No obstante, donde a nuestro juicio se puede constatar mejor los efectos del trabajo realizado fue en las subpruebas que valoraban el reconocimiento del nombre y del sonido de las letras. En ambos casos, se partía de un desconocimiento casi total del nombre de los grafemas y de cómo se pronuncia cada fonema, mientras que en la evaluación final las puntuaciones medias se incrementan notablemente, sobre todo en el reconocimiento del nombre de las letras, lo cual apunta a que los sujetos empiezan a establecer las relaciones fonema-grafema, aspecto que es un área de especial dificultad en los niños con TEL.

Al objeto de dar solidez a esos resultados, volvimos a pasar al grupo control algunas de las pruebas aplicadas en la evaluación inicial, efectuando también un contraste del rendimiento en la evaluación inicial y final. Dicho análisis reflejó lo siguiente:

TABLA VI

CONTRASTE DE LOS RESULTADOS DEL GRUPO CONTROL ANTES Y DESPUÉS DE LA INTERVENCIÓN (MEDIA, DESVIACIÓN TÍPICA Y PRUEBA DE WILCOXON)

\begin{tabular}{|c|c|c|c|}
\hline PRUEBAS & $\begin{array}{c}\text { EVALUACIÓN } \\
\text { INICIAL }\end{array}$ & EVALUACIÓN FINAL & $\begin{array}{c}\text { SIGNIFICATIVIDAD } \\
\text { PRUEBA DE WILCOXON }\end{array}$ \\
\hline $\begin{array}{c}\text { Peabody (Edad } \\
\text { Equivalente) }\end{array}$ & $\begin{array}{c}\mathrm{M}=2,43 \\
\mathrm{DT}=1,07\end{array}$ & $\begin{array}{c}\mathrm{M}=3,56 \\
\mathrm{DT}=0,72\end{array}$ & 0,10 \\
\hline Vocabulario (WPPSI) & $\begin{array}{c}\mathrm{M}=5,00 \\
\mathrm{DT}=4,58\end{array}$ & $\begin{array}{c}\mathrm{M}=8,00 \\
\mathrm{DT}=4,00\end{array}$ & 0,10 \\
\hline $\begin{array}{c}\text { Semejanzas (WPPSI) } \\
\text { Conciencia Fonológica } \\
\text { (p. directa) }\end{array}$ & $\begin{array}{c}\mathrm{M}=1,33 \\
\mathrm{DT}=1,15\end{array}$ & $\begin{array}{c}\mathrm{M}=7,66 \\
\mathrm{DT}=2,30\end{array}$ & 0,10 \\
\hline $\begin{array}{c}\text { Conciencia Fonológica } \\
\text { (p. centil) }\end{array}$ & $\begin{array}{c}\mathrm{MT}=15,66 \\
\mathrm{DT}=15,87\end{array}$ & $\begin{array}{c}\mathrm{M}=18,33 \\
\mathrm{DT}=26,55\end{array}$ & 0,15 \\
\hline $\begin{array}{c}\text { Procesamiento fonológico } \\
\text { (nivel silábico) }\end{array}$ & $\mathrm{M}=4,00$ & $\mathrm{MT}=28,83$ & 0,18 \\
\hline $\begin{array}{c}\text { Procesamiento fonológico } \\
\text { (nivel intrasilábico) }\end{array}$ & $\mathrm{DT}=5,29$ & $\mathrm{DT}=2,86,42$ & $\mathrm{MT}=8,00$ \\
\hline
\end{tabular}




\begin{tabular}{|c|c|c|c|}
\hline $\begin{array}{c}\text { Procesamiento fonológico } \\
\text { (nivel fonémico) }\end{array}$ & $\begin{array}{c}\mathrm{M}=0,66 \\
\mathrm{DT}=1,15\end{array}$ & $\begin{array}{c}\mathrm{M}=1,66 \\
\mathrm{DT}=1,52\end{array}$ & 0,31 \\
\hline Memoria fonológica & $\begin{array}{c}\mathrm{M}=13,66 \\
\mathrm{DT}=4,04\end{array}$ & $\begin{array}{c}\mathrm{M}=22,33 \\
\mathrm{DT}=11,54\end{array}$ & 0,28 \\
\hline $\begin{array}{c}\text { Reconocimiento nombre } \\
\text { letras }\end{array}$ & $\begin{array}{c}\mathrm{M}=12,50 \\
\mathrm{DT}=3,53\end{array}$ & $\begin{array}{c}\mathrm{M}=10,33 \\
\mathrm{DT}=9,60\end{array}$ & 0,18 \\
\hline $\begin{array}{c}\text { Reconocimiento sonido } \\
\text { letras }\end{array}$ & $\begin{array}{c}\mathrm{M}=0,00 \\
\mathrm{DT}=0,00\end{array}$ & $\begin{array}{c}\mathrm{M}=2,00 \\
\mathrm{DT}=9,60\end{array}$ & 0,31 \\
\hline
\end{tabular}

${ }^{*}=\mathrm{p} \leq 0.05$

A diferencia del grupo experimental, el grupo control no obtuvo progresos estadísticamente significativos en ninguno de los casos, lo cual nos permite interpretar que los avances del grupo experimental fueron debidos al trabajo realizado.

\section{DISCUSIÓNY CONCLUSIONES}

El estudio realizado ha podido demostrar que la elaboración de programas coherentes con los principios de la educación inclusiva puede resultar eficaz para responder a las necesidades singulares de los niños con dificultades del lenguaje como el TEL. No obstante, desde nuestro punto de vista, se hace necesario combinar las dos modalidades de trabajo; esto es, las del nivel 2 con las del nivel 3 para que estos sujetos saquen beneficios de la misma y empleen las habilidades adquiridas en situaciones naturales de interacción. Así, en el nivel 2 los niños con TEL han tenido muchas y ricas oportunidades para participar e interactuar en las actividades propuestas. Sin embargo, considerando el perfil inicial de los niños de la muestra, fue decisivo incluir también el nivel 3 de trabajo para conseguir los resultados ya comentados. Los niños con TEL difícilmente adquieren por sí mismos o con las actividades que habitualmente constituyen el currículo de Educación Infantil, las bases para acceder al aprendizaje lector (prueba de ello es la ausencia de progresos estadísticamente significativos en el grupo control). En este sentido, la constatación documentada de la influencia del lenguaje oral y las habilidades narrativas sobre la alfabetización y el éxito académico (Merrit y Culatta, 1998; Tabors, et al., 2001), tanto como de la conciencia y procesamiento fonológicos sobre el aprendizaje lector (Gimeno et al., 1994; Jiménez y Ortiz, 1995; Domínguez, 1996; Arnáiz Sánchez, et. al., 2001) eran razones poderosas para incorporar el nivel 3 de enseñanza, a sabiendas, por otra parte, de la influencia de las habilidades narrativas en la activación de otros aspectos del lenguaje, como el vocabulario y la complejidad gramatical. En este sentido, la naturaleza más clínica y personalizada de esta modalidad de enseñanza, basada en el recast conversacional y la práctica repetida en la que se apoyaba al niño a interiorizar y aplicar la estrategia de forma independiente, contribuyeron a que asimilaran y adquirieran un vocabulario más diverso y preciso y a que organizaran mejor sus emisiones, siendo más informativas. 
De la misma manera deben interpretarse los avances experimentados en la conciencia y procesamiento fonológicos. Las dificultades de los niños con TEL para el procesamiento del lenguaje, en general, pero sobre todo para las unidades menores del sistema lingüístico (sílabas y fonemas) requerían de un trabajo específico en una situación más controlada como la que se proporcionó en el nivel 3 de enseñanza. En este sentido, nuestros resultados coinciden con los de Guillon (2002; 2005), la cual documentó los posibles beneficios de exponer a los niños de Educación Infantil (4 y 5 años) al apoyo temprano en conciencia fonológica y en el conocimiento de las letras dentro del aula habitual, pero acompañada de una intervención explícita fuera del aula para facilitar la adquisición de las habilidades de conciencia silábica y fonémica, especialmente en escolares con TEL.

La modalidad de intervención adoptada en este trabajo, que combina los dos niveles, ya se ha utilizado en otros estudios, sobre todo cuando se quiere incidir en la efectividad de los tratamientos sobre objetivos de distintos componentes del lenguaje oral (Dollaghan, C., 2004; Leonard, et al., 2004)

Como conclusión final y respondiendo a los interrogantes planteados en este estudio, podemos afirmar que un trabajo inclusivo en los niveles 2 y 3 de intervención mejora ciertas habilidades de procesamiento fonológico y produce, asimismo, un aumento significativo en el caudal léxico y en la complejidad gramatical en sujetos con TEL, aspectos que contribuirán al progreso académico y social de este alumnado y, en última instancia a avanzar hacia su inclusión efectiva.

Somos conscientes, no obstante, de las limitaciones del estudio por lo que se refiere al tamaño limitado de la muestra, cuestión que se justifica por la dificultad para encontrar sujetos con TEL a edades tan tempranas. Habría que añadir, asimismo, que los estudios epidemiológicos muestran una prevalencia que oscila entre el 0,6 y el 7,4 por 100 , teniendo en cuenta, además, que muchas veces se incluyen en este grupo a niños y niñas con retraso de lenguaje.

Por último, creemos oportuno subrayar que con este trabajo no se está buscando exclusivamente una relación causa-efecto entre la intervención y la mejora de los niños, para ello hubiera sido más idóneo otro tipo de diseño.Nuestro propósito es planificar acciones innovadoras, dentro del aula, con la colaboración entre profesionales y sin desmantelar continuamente los contextos naturales, acompañando esta práctica de las oportunas evaluaciones periódicas que nos permitan introducir cambios y mejoras

\section{REFERENCIAS BIBLIOGRÁFICAS}

Acosta, V., Moreno, A., Barroso, M., Coello, A. y Mesa, J. (2007). Guía de actuaciones educativas en el ámbito de la comunicación y el lenguaje. Consejería de Educación, Cultura y Deportes. Gobierno de Canarias.

Acosta, V., Moreno, A., Axpe, M. y Quintana, A. (2008). La identificación de barreras para el aprendizaje de la lectura entre alumnado de riesgo desde una perspectiva integrada. Revista de Logopedia, Foniatría y Audiología, 28, 4, 231-244.

Acosta, V., Moreno, A. y Axpe, M. ( $a$, en prensa). Intervención sobre la conciencia fonológica en sujetos con trastorno específico del lenguaje en contextos inclusivos: posibilidades y limitaciones. Bordón, aceptado para su publicación. 
Acosta, V., Moreno, A. y Axpe, M. (b, en prensa). La intervención inclusiva para la mejora de habilidades de lenguaje oral y de lectura inicial en niños con trastorno específico del lenguaje. Revista de Educación, aceptado para su publicación.

Aguado, G. (2007). Repetición de pseudopalabras en el diagnóstico del Trastorno Específico del Lenguaje. Navarra, Universidad de Navarra.

Aguilar, E. y Serra, M. (2003). A-RE-HA. Análisis del retraso del habla. Barcelona, Edicions Universitat de Barcelona.

Aguinaga, G., Armentia, M., Fraile, A., Olangua, P. y Útiz, N. (2004). Prueba de Lenguaje Oral de Navarra-revisada (PLON-R). Pamplona, Fondo de Publicaciones del Gobierno de Navarra.

Arnaiz, P., Castejón, L. y Ruiz, Ma S. (2001). Influencia de un programa de desarrollo de las habilidades psicolingüísticas en el acceso a la lecto-escritura. Investigación Educativa, 20 (1), 189-208.

Bradley, R., Danielson, L., y Doolittle, J. (2005). Response to intervention. Journal of Learning Disabilities, 38, 485-486.

Camacho, J. (1994). Manual de uso del programa estadístico spss/pc+. Barcelona, PPU

Cardona, $\mathrm{M}^{\mathrm{a}}$ C. (2003). Inclusión y cambios en el aula vía adaptaciones instructivas. Investigación Educativa, 21 (2), 465-487.

Coleman, M., Buysse, V. y Neitzel, J. (2006). Recognition and response: an early intervening system for young children at risk for learning disabilities. Full report. Chapel hill: the University of North Carolina at chapel hill, fpg child development institute.

Conti-Ramsden, G. y Botting, N. (2000). Educational placements for children with Specific Language Impairments. En D.V.M. Bishop y L.B. Leonard (eds.) speech and language impairments in children. Causes, characteristics, intervention and outcome. Hove, Psychology Press.

Domínguez, A. (1996). El desarrollo de habilidades de análisis fonológico a través de programas de enseñanza. Infancia y Aprendizaje, 76, 69-81.

Dollaghan, C. (2004). Evidence-based practice in communications disorders: what do we know and when do we know it? Journal Communication Disorder, 37, 391-400

Dunn, L., Padilla, E., Lugo, D. y Dunn, L. (1986). Test de vocabulario en imágenes Peabody. Madrid, TEA.

Fernández, A., Machuca, J. y García, L. (2001). Hacia una diagnóstico comprensivo de diagnósticos inespecíficos de alumnos con dificultades para el aprendizaje escolar. Investigación Educativa, 19 (1), 63-82.

Fuchs, D., Fuchs, L. (2006). Introduction to response to intervention: what, why, and how valid is it? Reading Research Quarterly, 41, 1, 93-99.

Guillon, G. (2002). Follow-up study investigating benefits of phonological awareness intervention for children with spoken language impairment. International Journal of Language and Communication Disorders, 37, 4, 381-400.

Guillon, G. (2005). Facilitating phoneme awaresness development in 3 and 4 year old children with speech impairment. Language, Speech, and Hearing Services in Schools, 36, 308-324.

Gimeno, A., Clemente, A., López, T. y Castro, A. (1994). ¿Cómo mejorar la conciencia fonológica en la escuela? Descripción y análisis de un programa de intervención. CLEE, 21, 87-95. 
Jiménez, J. y Ortiz, R. (1995). Conciencia fonológica y aprendizaje de la lectura: teoría, evaluación e intervención. Madrid, Síntesis.

Juárez, A. y Monfort, M. (1996). Registro Fonológico Inducido. Madrid, CEPE.

Justice, L. (2006). Evidence-based practice, response-to-intervention and prevention of reading difficulties. Language, Speech, and Hearing Services in Schools, 37, 1-14.

Kirk, S., McCarthy, J. y Kirk, W. (1968). Test Illinois de Habilidades Psicolingüísticas (ITPA). Madrid, TEA.

Lara, M. Aguilar, E. y Serra, M. (2007). Prueba de Procesamiento Fonológico (PROFON). Colombia, Universidad Nacional de Colombia.

Leonard, L., Camarata, S., Brown, B., Camarata, M. (2004). Tense and agreement in the speech of children with specific language impairment. Journal Speech Language Hearing Research, 47, 1363-79.

McCarthy, D. (1976). Escalas McCarthy de aptitudes y psicomotricidad para niños. Madrid, TEA.

Merrit, D. y Culatta, B. (1998). Language intervention in the classroom. San Diego, CA, Singular Publishing Group.

Miller, J. F (1991). Quantifying productive language disorders. En J.F Miller (ed.), Research on child language disorder. A decada of progress. Austin, TX, Pro-ed (pp. 211-220).

Newborg, J., Stock, J. y Weck, L. (2001). Inventario de Desarrollo Battelle. Madrid, TEA.

O'Connor, R., Fulmer, D., Harthy, K. y Bell, K. (2005). Layers of reading intervention in kindergarten through third grade: changes in teaching and students outcomes. Journal of Learning Disabilities, 38, 440-455.

Pavez, M., Coloma, C. y Maggiolo, M. (2008). El desarrollo narrativo en niños. Barcelona, Ars Médica.

Peggy, R. (2001). Buenas noches, gorila. Nueva York, Putnan's.

Tabors, P, Snow, C. y Dickinson, D. (2001). Homes and schools together: supporting language and literacy development. In Dickinson D, Tabors P, eds. Beginning literacy with language. Baltimore, Brookes (pp. 313-34).

Tomblin, J, Records, N, Buckwalter, P, Zhang, X, Smith, E, O'brien, M. (1997). Prevalence of specific language impairment in kindergarten children. Journal Speech Language Hearing Research. 40, 1245-1260.

Vaughn, S., Liann-Thompson, S. y Hickman, P. (2003). Response to instruction as a means of identifying students with reading/learning disabilities. Exceptional Children, 69, 391-409.

Wechler, D. (1970). Test de Inteligencia para Preescolares (WPSSI). Madrid, TEA.

Zimmerman, I., Steiner, V. y Pond, R. (2003). Preschool Language Scale-Spanish-4(PLS-4). Chicago, Psychological Corporation.

Fecha de recepción: 17 de diciembre de 2010.

Fecha de revisión: 05 de febrero de 2011.

Fecha de aceptación: 17 de marzo de 2011. 